\title{
Assessing Ecological Risks at the Landscape Scale: Opportunities and Technical Limitations
}

\author{
Lawrence A. Kapustka $^{1}$
}

\begin{abstract}
There is a growing awareness that ecological risk assessments (ERAs) could be improved if they made better use of ecological information. In particular, landscape features that determine the quality of wildlife habitat can have a profound influence on the estimated exposure to stressors incurred by animals when they occupy a particular area. Various approaches to characterizing the quality of habitat for a given species have existed for some time. These approaches fall into three generalized categories: (1) entirely qualitative as in suitable or unsuitable, (2) semiquantitative as in formalized habitat suitability index models, or (3) highly quantitative site-specific characterization of population demographic data such as matrix population models or multiple regression models. Such information can be used to generate spatially explicit estimates of exposure to chemicals or other environmental stressors, e.g., invasive species, physical perturbation, that take into account the magnitude of co-occurrence of the animals and stressors as they forage across a landscape. In this way, greater ecological realism is provided in the ERA and more informed management decisions can be attained.
\end{abstract}

Key Words: landscape ecology; wildlife habitat characterization; habitat suitability index models; ecological risk assessments

\section{INTRODUCTION}

Plants and wildlife are subjected to many environmental stressors, including the diverse array of biocides and industrial chemicals in the environment (Ferenc and Foran 2000, DorwardKing et al. 2001). Invasive species and physical perturbations also have major effects on ecological system functions. The ecological risk assessment (ERA) process (EPA 1998) has been an effective tool to evaluate the effects of stressors on ecological resources. However, concerns have been raised about the usefulness of ERAs as generally practiced to help support environmental management decisions (Fairbrother et al. 1995, 1997, Tannenbaum 2002). Broadening the focus of ERAs to include quality of habitat is especially important because potentially adverse effects on wildlife populations are not limited to chemical effects.
Animal use of a particular area varies in relation to cues in the landscape that signal the availability of food, shelter, or other resources favored by the particular species. This use often fluctuates seasonally and changes at different life-history stages. Individuals or groups of individuals are drawn to food and suitable physical structures, and avoid areas of lower quality. The term habitat, although often used loosely as an indication of environmental quality, refers to the combination of physical and biological features preferred by a particular species. Different habitat preferences reflect the evolution and adaptation of diverging species (Whittaker 1975). Some species are attracted to disturbance zones and edges, but others avoid such areas. Regarding ERAs, to the extent that fish and wildlife respond to differences in landscape features, e.g., attraction, avoidance, habitat quality will determine which and how many receptors or assessment species will be exposed. Spatial relationships, stressors, and foraging activities influence exposure: co-located distributions 
increase exposure, whereas disjoint distributions decrease exposure.

Several groups have described generalized ways to consider habitat quality in ERAs. Rand and Newman (1998) suggested using habitat suitability index (HSI) models in ERAs, but provide no examples of their use and do not give specific details on how to integrate habitat information with exposure assessment or risk characterization. Freshman and Menzie (1996) described two approaches that take into account spatial differences in contaminant concentrations with respect to foraging activities and the proportion of a local population likely to be exposed to the contaminants. Hope (2000, 2001, 2004), Wickwire et al. (2004), and Linkov et al. (2001, 2002, 2004a) have used placeholder habitat values to illustrate the effect of habitat on cumulative exposure levels. Kapustka et al. (2001, 2004) described procedures to use HSIs as the habitat quality parameter for estimating exposure levels. Most recently, the American Society for Testing and Materials (ASTM) published a Standard Guide for Estimating Wildlife Exposure Using Measures of Habitat Quality (ASTM 2005). In this paper, I review efforts to implement the use of habitat considerations in spatially explicit ERAs.

\section{APPROACH}

\section{Ecological risk assessment framework for considering habitat information}

The U.S. Environmental Protection Agency (EPA) process for conducting ecological risk assessments (ERAs) promotes a tiered approach that emphasizes the importance of site-specific, ecologically relevant information (EPA 1998, 2001). It begins with problem formulation and screening-level risk analysis using point estimates and continues toward more complex probabilistic analyses. The EPA (1998) acknowledged the importance of considering habitat when evaluating risk; however, no specific methods were proposed for doing so. During problem formulation, several decisions regarding the selection of assessment species, i.e., receptors and end points, provide the ultimate focus of the ERA. Traditionally, the assessment species expected to have the maximum exposure because of characteristics such as dietary preferences, foraging behavior, and residency, plus sensitivity to chemicals of potential concern (CoPC), have been chosen to serve as surrogates for the collection of organisms at a site. This often tends to favor the inclusion of species that have restricted foraging ranges, especially if they are among the charismatic megafauna. Modification of the EPA process to address spatially explicit details was discussed in Kapustka et al. (2001) and appears in the ASTM standard (ASTM 2005). The approach focuses on improving decision-making relevant to the Remedial Investigation/Feasibility Study (RI/FS) process. The intermediate and final tiers may include spatially explicit and population-based risk assessments. A spatially explicit approach identifies the specific areas that contribute the most to exposure estimates and risk. If unacceptable levels of risk are demonstrated, different scenarios for cleanup or other mitigation strategies, including active management of habitat quality, can be evaluated. Kapustka et al. (2001) identified five of 12 scenarios in which habitat considerations in ERAs can be useful, i.e., where the landscape or the concentrations of CoPC is heterogeneous, in conducting spatially explicit ERAs.

\section{Alternative approaches to characterizing habitat quality}

Habitat characteristics for a particular species are determined by landscape features such as vegetation cover, the availability of food items, physical components, etc. The size of the site relative to the home range or foraging range of individuals of a species should also be considered in assessing the potential value of habitat characterization. Alhough precise areas are elusive, intuitively there is some minimum area required before habitat characterization is warranted. The de minimus area may also differ between resident species and migratory species (Fig. 1).

There are different ways to characterize habitat quality that range in levels of sophistication ( Fig. 2 ). Very broadly defined characterization such as binary, e.g., suitable vs. unsuitable, or trinary, e.g., good, bad, or ugly, may suffice for some situations. If greater rigor in characterizing habitat is warranted, then the choice may be to use the semiquantitative structure provided in habitat suitability index (HSI) models. Alternatively, detailed site characterization of population density and structure for use in population matrix models or various multiple regression models may be 
Fig. 1. Generalized relationship of the relevance of characterizing habitat quality in an ecological risk assessment vs. the size of the site, taking into account the home range and residency status of the assessment species. $A_{r}$ represents a resident species with a small range; $A_{m}$, a migrant species with a small range; $B_{r}$, a resident species with a large range; and $\mathrm{B}_{\mathrm{m}}$, a migrant species with a large range.

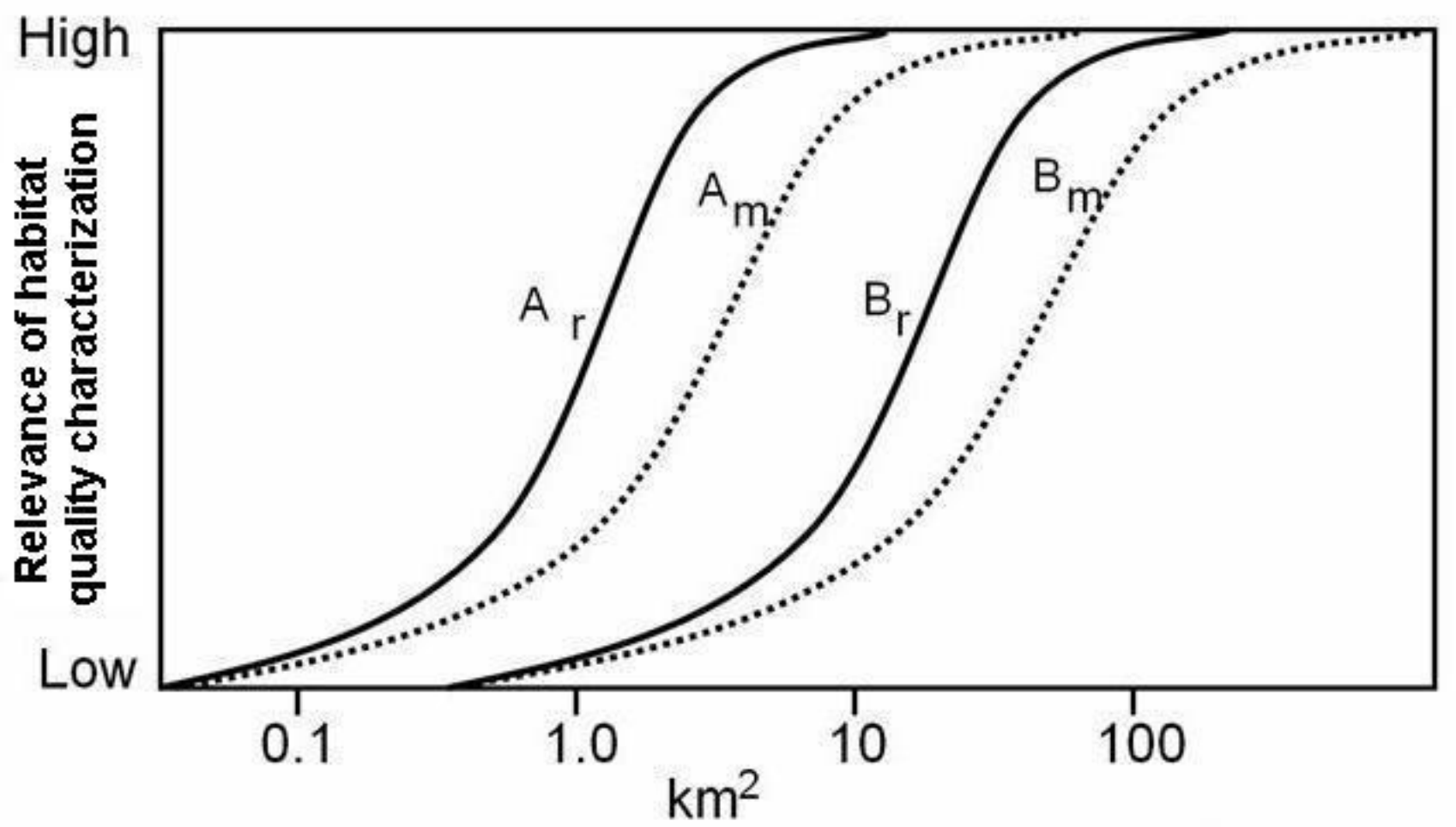

appropriate. Criteria for selecting the appropriate method to characterize habitat from among these different levels of sophistication should be established during problem formulation.

\section{Habitat suitability index database}

HSI models have been developed for many species of interest. The U.S. Fish and Wildlife Service formalized the characterization of habitat for certain species in the 1990s (Schroeder and Haire 1993). Currently, there are more than 160 HSI models published, although quantitative predictions of population densities are limited (Terrell and Carpenter 1997).

Information from 90 HSI models was abstracted into an Access ${ }^{\circledR}$ database and Excel ${ }^{\circledR}$ workbooks (Kapustka et al. 2004). This information includes descriptions of all the variables required to calculate HSI values, areas of applicability, qualitative sensitivity analyses, and suggested methods available to obtain field data. The Excel $^{\circledR}$ workbooks contain executable equations for each of the models, which convert the raw data from each variable into the corresponding HSI value. 
Fig. 2. Decision tree for determining the applicability of habitat characterization and the level of sophistication warranted in assessing habitat quality. EcoRA stands for ecological risk assessment.

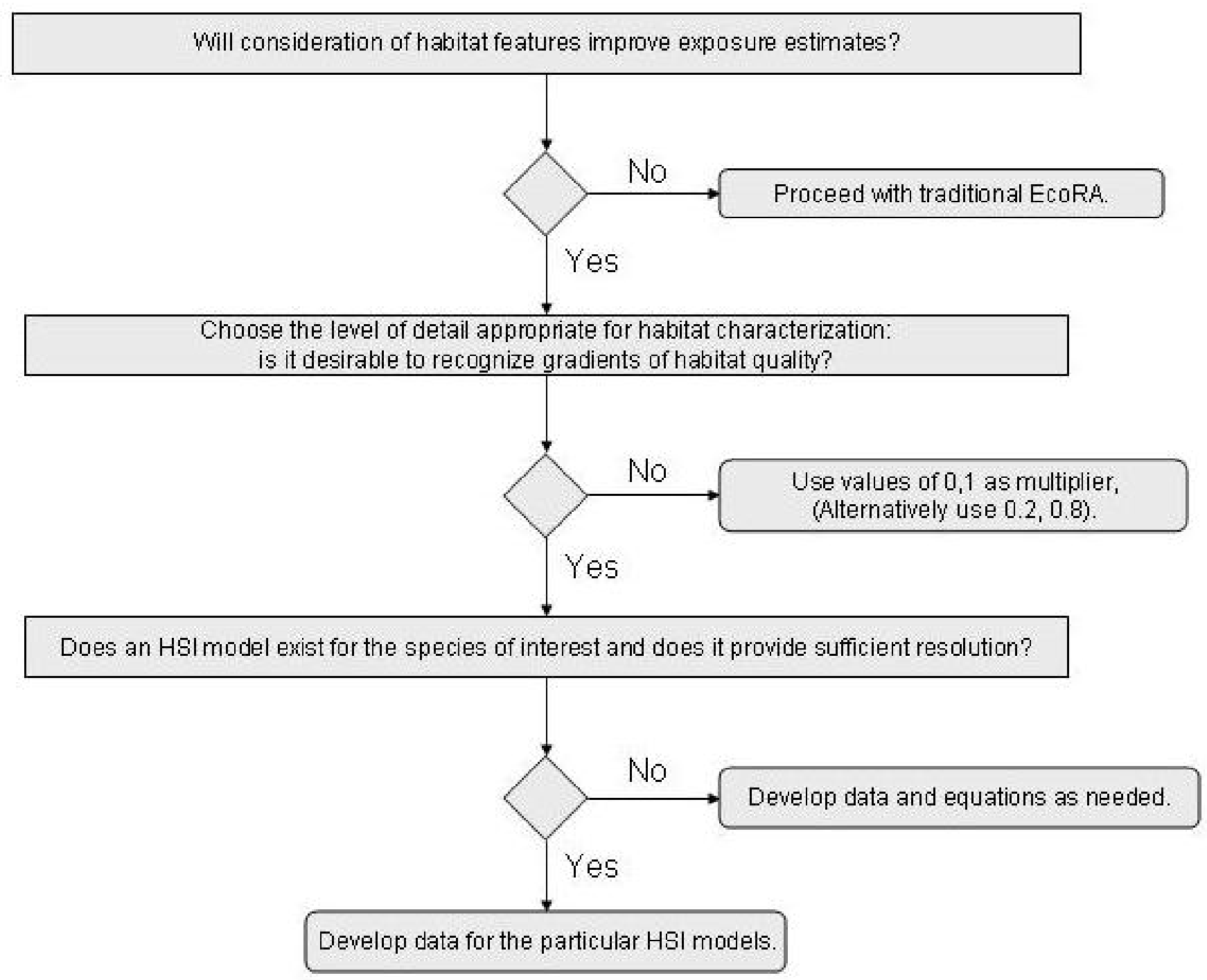

Kapustka et al. (2001, 2004) described the selection of assessment species based on an examination of available HSI models and exposure information. Once the candidate assessment species have been identified, the list of HSI models can be used to develop the sampling plan. The list of HSI species is used to query the database; the resulting database report provides a compiled list of all the variables needed to calculate HSI models for all the selected species.The majority of the variables used in terrestrial and wetland HSI models are measurements of vegetation or other landscapelevel features. These include parameters such as percentage canopy cover; distance between cover types, e.g., forest edge to water, forest patch to forest patch; or other features that can be acquired from aerial imagery. Others require on-site determination of variables related to the structure of the plant community, such as height of shrub canopy, percentage herbaceous cover under a forest canopy, 
size-class distribution of trees, etc. Others require detailed quantification of variables related to the structure of the plant community, such as the number of nesting cavities in large trees. Methods for acquiring such data are described in the E1923-97 Standard Guide for Sampling Terrestrial and Wetlands Vegetation (ASTM 1998).

Examination of particular HSI equations also reveals differences in variable sensitivity. Qualitative sensitivity features of the models have been coded in a comment field in the database. For scoping- or screening-level assessments, estimates of variables that are particularly difficult to parameterize can provide a rapid, preliminary indication of the importance of gathering particular data. By examining the list of variables, weighing the preferred and alternative methods that may be used to generate the required data, and reviewing the sensitivity of the variables, those variables that can be satisfied using aerial images, routine on-site survey methods, and specialized or detailed on-site survey procedures can be rapidly identified. From this, it is a relatively straightforward process to devise a progressive sampling plan from reconnaissance-level through definitive-level ERAs that maximizes the number of models satisfied with different levels of sampling effort. Such a plan can also be used to produce a financial risk assessment for a project, i.e., identifying the benefits of obtaining all the information at once vs. deferring certain data collection procedures to later stages of risk assessment.

\section{Spatially explicit modeling of exposure}

Linkov et al. (2004b) incorporated HSI model values into a prototype software program that generates spatially explicit exposure estimates in which information on habitat quality is used to modulate the magnitude of exposure. Modeled foraging movement across the site is determined by the relative attractiveness of the habitat in each polygon as reflected by the species-specific assigned HSI value.

After the delineation of polygons, each polygon is assigned a value for habitat quality and a value for CoPC concentration. Currently, the program operates as a stand-alone feature. User-provided digital images such as maps or photos are used for the orientation and display of polygons, but these are not linked to data tables. In the future, the program will be incorporated into a GIS framework. The user will be able to develop scenarios and specify model parameters including the input of maps or shape files to identify polygons based on landscape features. The polygons can then be assigned values for habitat quality, for the species of interest, and stressor levels, which in current versions are chemical concentrations.

In Risk Trace, the HSI module uses Microsoft Excel functions and Visual Basic (Reference) to calculate the HSI of a specified polygon that reflects the quality and the suitability of habitat in this polygon for a selected species. The HSI model considers easily quantified environmental features to parameterize linear models of the following form:

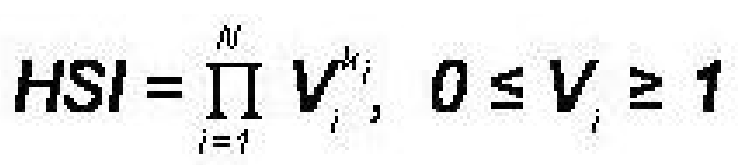

The HSI value determines the relationships between species or a group of species and critical landscape features that define quality of habitat. The model uses landscape features and structures, i.e., dozens of variables $\left(\mathrm{V}_{\mathrm{i}}\right)$, from each specified polygon and returns a relative HSI value ranging from 0 (unsuitable conditions) to 1 (ideal conditions).

Risk-Trace calculates receptor exposures and risks. The appropriate calculation algorithms are automatically selected depending on how a user describes specific scenarios via the interface. The modules include the following submodules:

\section{Probabilistic receptor migration model}

This simulates a receptor's stochastic movement across the landscape based on attractiveness, diet preferences, and availability of forage as captured in the surrogate measures of habitat quality, i.e., HSI. The model uses receptor velocity, forage volume, and habitat quality (HSI), and returns relative duration of presence in different zones of migration. 


\section{Spatially explicit exposure assessment model}

This compiles dietary exposure levels from feeding in the areas of different contamination and determines time-dependent contaminant accumulation in receptor tissue using a differential balance equation and an equation of continuity of concentrations. The model uses consumption and contamination of forage and returns dose of exposure. Note that user input of variables is needed for each substance of interest. However, in most cases, dietary exposure will dominate the assessment if dermal or inhalation exposures are deemed important; these could easily be added to the assessment.

\section{Screening-level risk assessment model}

This model calculates a point estimate risk quotient (RQ) or hazard quotient (HQ) for each contaminant; these are equal to the site contaminant concentration divided by the selected benchmark concentration for ecological receptors. These RQs and HQs can be considered threshold references values.

The database module uses Microsoft Access as a data management platform. The libraries of receptor characteristics, e.g., body weight, habitat size, diet, threats, etc., are stored in separate tables. The HSI variable libraries include those characteristics of habitat and landscape as well as their relationships, which generically define quality and suitability of polygon environment for vital and reproduction functions of the wildlife populations.

The prototype software was tested and debugged using Microsoft Windows (1995, 1998, 2000, and $\mathrm{XP}$ versions). Tests of the software were performed for the following scenarios:

- Estimation of exposure and risk for spatially homogeneous contamination, characterized by the averaged concentrations of toxic substances, i.e., receptor migration is not taken into consideration, and exposure is modeled deterministically.

- Estimation of exposure and risk, with receptor migration modeled probabilistically depending on forage attractiveness and other factors discussed above.

\section{DISCUSSION}

Many regions are characterized by the frequent occurrence of adverse anthropogenic stressors. These territories may constitute areas with high biodiversity and contain habitats that are potentially valuable for some endangered species. For such areas, decisions regarding site-specific land use planning and remediation alternatives are based on a variety of factors. A systematic and modular framework that makes it possible to weigh the relative importance of these factors would be a valuable tool to determine appropriate alternatives for specific sites. Within this framework, risk for wildlife from exposures and habitat disturbances resulting from human activity is a governing factor.

The carrying capacity of the environment, habitat quality and suitability, and landscape contamination and disturbance are characterized by different spatial patterns and temporal scales. These factors result in different behaviors and foraging strategies among receptors; as a result, different species may experience significantly different chemical exposures on the same site, even if their foraging strategies overlap. Currently, exposure estimates and subsequent human health and ecological risk projections usually assume a static and continuous exposure of an ecological receptor to a contaminant concentration represented by some descriptive statistic, such as the mean or maximum. These assumptions are generally overly conservative and ignore some of the major advantages offered by risk assessment, i.e., the ability to account for sitespecific conditions and to conduct iterative analyses.

The suite of species used for the ecological risk assessment (ERA) ultimately must be assessable, $\mathrm{i}$. e., data must be available to calculate or infer exposure to and effects of stressors. Frequently, surrogate species are used to represent broadly defined groups of potentially important species that are categorized primarily by trophic levels, e.g., a mammalian herbivore, an avian insectivore, or a top carnivore. The ecological relevance of the species used in the assessment is frequently a contentious point of debate. However, monetary constraints typically limit the opportunities to reassess the use of more relevant species. If a broader suite of species were considered assessment species at the outset, such arguments could be avoided. To do so requires a structured approach that takes into account all the potential species at a site and documents in the 
administrative records the rationale for narrowing the species list to a manageable number.

The central premise of this approach is that the highest-quality ERAs are those that focus on assessment species for which wildlife habitat requirements, preferences, and exposure factors, e. g., dietary preferences, feeding rates, metabolic rates, etc., are known. To achieve a high-quality ERA using species for which such information is missing requires considerable commitment of time and money to obtain the requisite data. High-profile sites, i.e., sites of great interest to the public or with important societal consequences, may warrant the expenditures related to information gathering. However, for most sites, the collection of basic biological or ecological data is beyond consideration. In such situations, a transparent process could facilitate communication among stakeholders and improve acceptance of the risk assessment.

Habitat suitability index (HSI) models provide a controlled and economical means of accounting for habitat conditions. The qualitative differences that occur in landscape features under various remediation scenarios provide broad characterization of the effects in terms of habitat quality for the wildlife species of interest. When viewed in relative terms, rather than by the absolute quantification of species abundance, the HSI model output can be a valuable tool in ERA (Kapustka et al. 2003).

Land-use patterns have been described as the most critical aspect affecting wildlife populations and regional biodiversity (Turner et al. 2001). Chemical contamination often ranks low in terms of the many factors limiting wildlife populations. Regulatory and legislative efforts in the United States have begun to promote "brownfield development" as an alternative to expansion into uncontaminated areas and with less stringent cleanup standards. Until recently, many areas with low- to moderate-levels of chemical contamination were nevertheless subjected to intrusive remediation efforts, resulting in substantial destruction of existing wildlife habitat and low potential for enhancing habitat quality at the affected site.

Landscape relationships are being considered in an EPA program designed to incorporate ecological dynamics into risk assessments. The Program to Assist in Tracking Critical Habitat model uses a GIS platform that allows user input in defining polygons and their characteristics (Schumaker 1998; www.epa.gov/wed/pages/models.htm). The Army Risk Assessment Modeling System ( www.wes.army.mil/el/arams/arams.html) is developing models that use habitat quality assessments to improve the realism of exposure assessments. Akçakaya et al. (2004) edited a collection of papers involving 37 species for which spatially explicit ecological parameters related to populations and metapopulation dynamics were evaluated to support management decisions. The tools to conduct the computational steps and develop information displays such as graphs, maps, and charts are becoming increasingly sophisticated and userfriendly. These advances are particularly encouraging in the thrust to expand ecological risk assessment to include biological and physical stressors along with the traditional chemocentric approaches. In general, landscape information can be used to generate spatially explicit exposure estimates using habitat quality information to modulate the magnitude of exposure (Kapustka et al. 2001, 2004). Each of these efforts seeks to capture landscape information and place it in a framework that can inform risk assessors and risk managers.

Responses to this article can be read online at: http://www.ecologyandsociety.org/vollo/iss $2 /$ art11/responses/

\section{Acknowledgments:}

Partial funding for this effort was provided through the American Chemistry Council Long-Range Research Initiative-Sponsored Research Program, Grant Number EEE0015.

\section{LITERATURE CITED}

Akçakaya, H. R., M. Burgman, O. Kindvall, C. C. Wood, P. Sjögren-Gulve, J. S. Hatfield, and M. A. McCarthy. 2004. Species conservation and management: case studies. Oxford University Press, Cary, North Carolina, USA.

American Society for Testing and Materials (ASTM). 1998. Standard guide for sampling 
terrestrial and wetlands vegetation. ATSM Annual Book of Standards, E1963-98, Volume 11.05. ASTM, Consohocken, Pennsylvania, USA.

American Society for Testing and Materials (ASTM). 2005. Standard guide for estimating wildlife exposure using measures of habitat quality. ATSM, E1963-98, Volume 11.05. ASTM, Consohocken, Pennsylvania, USA.

Dorward-King, E. J., G. W. Suter, L. A. Kapustka, D. R. Mount, D. K. Reed-Judkins, S. M. Cormier, S. D. Dyer, M. G. Luxon, R. Parrish, and G. A. Burton. 2001. Distinguishing among factors that influence ecosystems. Pages 1-26 in D. J. Baird and G. A. Burton, editors. Ecological variability: separating natural from anthropogenic causes of ecosystem impairment. SETAC Press, Pensacola, Florida, USA.

Fairbrother, A., L. A. Kapustka, B. A. Williams, and J. Glicken. 1995. Risk assessment in practice: success and failure. Human Ecological Risk Assessment 1:367-375.

Fairbrother, A., L. A. Kapustka, B. A. Williams, and R. S. Bennett. 1997. Effects-initiated assessments are not risk assessments. Human Ecological Risk Assessment 3:119-124.

Ferenc S. A., and J. A. Foran. 2000. Multiple stressors in ecological risk and impact assessment: approaches to risk estimation. SETAC Press, Pensacola, Florida, USA.

Freshman J. S., and C. A. Menzie. 1996. Two wildlife exposure models to assess impacts at the individual and population levels and the efficacy of removal actions. Human Ecological Risk Assessment 2:481-498.

Hope B. 2000. Generating probabilistic spatiallyexplicit individual and population exposure estimates for ecological risk assessments. Risk Analysis 20:573-589.

Hope, B. 2001. Consideration of bioenergetic factors in spatially-explicit assessments of ecological receptor exposure to contaminants. Toxicology and Industrial Health 17:322-332.

Hope, B. 2004. A multi-stressor terrestrial ecological exposure model. Pages 311-323 in L. A. Kapustka, H. Galbraith, M. Luxon, and G. R.
Biddinger, editors. Landscape ecology and wildlife habitat evaluation: critical information for ecological risk assessment, land-use management activities, and biodiversity enhancement practices. ASTM STP 1458. American Society for Testing and Materials International, West Conshohocken, Pennsylvania, USA.

Kapustka, L.A. 2003. Rationale for use of wildlife habitat characterization to improve relevance of ecological risk assessments. Human Ecological Risk Assessment 9:1425-1430.

Kapustka L. A., H. Galbraith, and M. Luxon. 2001. Using landscape ecology to focus ecological risk assessment and guide risk management decision-making. Toxicology and Industrial Health 17:236-246.

Kapustka L. A., H. Galbraith, M. Luxon, J. Yocum, and B. Adams. 2004. Application of habitat suitability index values to modify exposure estimates in characterizing ecological risk. Pages 169-194 in L. A. Kapustka, H. Galbraith, M. Luxon, and G. R. Biddinger, editors. Landscape ecology and wildlife habitat evaluation: critical information for ecological risk assessment, land-use management activities, and biodiversity enhancement practices. ASTM STP 1458. American Society for Testing and Materials International, West Conshohocken, Pennsylvania, USA.

Linkov, I., D. Burmistrov, J. Cura, and T. S. Bridges. 2002. Risk-based management of contaminated sediments: consideration of spatial and temporal patterns of exposure modeling. Environmental Science and Technology 36:238-246.

Linkov, I., A. Grebenkov, A. Andrizhievski, A. Loukashevich, and A. Trifonov. 2004a. Risktrace: software for spatially explicit exposure assessment. Pages 286-296 in L. A. Kapustka, H. Galbraith, M. Luxon, and G. R. Biddinger, editors. Landscape ecology and wildlife habitat evaluation: critical information for ecological risk assessment, land-use management activities, and biodiversity enhancement practices. ASTM STP 1458. American Society for Testing and Materials International, West Conshohocken, Pennsylvania, USA.

Linkov, I., A. Grebenkov, and V. M. Baitchorov. 2001. Spatially explicit exposure models: application to military sites. Toxicology and 
Industrial Health 17:230-235.

Linkov, I., L. A. Kapustka, A. Grebenkov, A. Andrizhievski, A. Loukashevich, and A. Trifono. $2004 b$. Incorporating habitat characterization into Risk-trace: software for spatially explicit exposure assessment. Pages 253-265 in I. Linkov, and A. Ramadan, editors. Comparative risk assessment and environmental decision making. Kluwer Academic, Dordrecht, The Netherlands.

Rand, G. M., and J. R. Newman. The applicability of habitat evaluation methodologies in ecological risk assessment. Human Ecological Risk Assessment 4:905-929.

Schroeder, R. L., and S. L. Haire. 1993. Guidelines for the development of community-level habitat evaluation models. Biological Report Number 8. U.S. Department of the Interior, U.S. Fish and Wildlife Service, Washington, D.C., USA.

Shumaker, N. H. 1998. A users guide to the PATCH model. EPA/600/R-98/135. Environmental Research Laboratory, U.S. Environmental Protection Agency, Corvallis, Oregon, USA.

Tannenbaum, L. V. 2002. Terrestrial ecological risk assessment: are we missing the forest for the trees? SETAC Globe 3(4):38-39.

Terrell, J. W., and J. Carpenter. 1997. Selected habitat suitability index model evaluations. USGS/ BRD/ITR 1997-0005. U.S. Department of the Interior, U.S. Geological Survey, Washington, D. C., USA.

Turner M. G., R. H. Gardner, and R. V. O'Neill. 2001. Landscape ecology in theory and practice, patterns and process. Springer-Verlag, New York, New York, USA.

U.S. Environmental Protection Agency (EPA). 2001. Risk assessment guidance for superfund. Volume III, Part A: process for conducting probabilistic risk assessment. EPA 540-R-02-002, OSWER 9285.7-45. EPA, Washington, D.C., USA.

U.S. Environmental Protection Agency (EPA). 1998. Guidelines for ecological risk assessment. EPA/630/R095/002F. Risk Assessment Forum, Washington, D.C., USA.

Whittaker, R. H., editor. 1975. Communities and ecosystems. Second edition. MacMillan, New York, New York, USA.

Wickwire W. T., C. A. Menzie, D. Burmistrov, and B. K. Hope. 2004. Incorporating spatial data into ecological risk assessments: spatially explicit exposure module SEEM for ARAMS. Pages 297-310 in L. A. Kapustka, H. Galbraith, M. Luxon, and G. R. Biddinger. editors. Landscape ecology and wildlife habitat evaluation: critical information for ecological risk assessment, land-use management activities, and biodiversity enhancement practices. ASTM STP 1458. American Society for Testing and Materials International, West Conshohocken, Pennsylvania, USA. 